\title{
ARTICLE \\ Clinical Study \\ Safety and pharmacokinetics of MM-302, a HER2-targeted antibody-liposomal doxorubicin conjugate, in patients with advanced HER2-positive breast cancer: a phase 1 dose-escalation study
}

\author{
Pamela Munster ${ }^{1}$, Ian E. Krop ${ }^{2}$, Patricia LoRusso ${ }^{3}$, Cynthia Ma $^{4}$, Barry A. Siegel ${ }^{5}$, Anthony F. Shields ${ }^{6}$, István Molnár ${ }^{7}$, \\ Thomas J. Wickham ${ }^{7}$, Joseph Reynolds ${ }^{7}$, Karen Campbell ${ }^{7}$, Bart S. Hendriks ${ }^{7}$, Bambang S. Adiwijaya ${ }^{7}$, Elena Geretti ${ }^{7}$, \\ Victor Moyo ${ }^{7}$ and Kathy D. Miller ${ }^{8}$
}

BACKGROUND: This phase 1 dose-escalation trial studied MM-302, a novel HER2-targeted PEGylated antibody-liposomal doxorubicin conjugate, in HER2-positive locally advanced/metastatic breast cancer.

METHODS: Patients were enrolled in four cohorts: MM-302 monotherapy $\left(8,16,30,40\right.$, and $50 \mathrm{mg} / \mathrm{m}^{2}$ every 4 weeks [q4w]); MM-302 (30 or $40 \mathrm{mg} / \mathrm{m}^{2} \mathrm{q} 4 \mathrm{w}$ ) plus trastuzumab (4 mg/kg q2w); MM-302 (30 mg/m²) plus trastuzumab (6 mg/kg) q3w; MM-302 (30 $\left.\mathrm{mg} / \mathrm{m}^{2}\right)$ plus trastuzumab $(6 \mathrm{mg} / \mathrm{kg})$ and cyclophosphamide $\left(450 \mathrm{mg} / \mathrm{m}^{2}\right) \mathrm{q} 3 \mathrm{w}$.

RESULTS: Sixty-nine patients were treated. The most common adverse events (AEs) were fatigue and nausea. Grade $3 / 4$ AEs of special interest included neutropenia, fatigue, mucosal inflammation, anemia, thrombocytopenia, febrile neutropenia, and palmarplantar erythrodysesthesia. The MTD was not reached. With MM-302 $\geq 30 \mathrm{mg} / \mathrm{m}^{2}$, overall response rate (ORR) was $13 \%$ and median progression-free survival (mPFS) 7.4 months (95\% Cl: 3.5-10.9) in all arms. In 25 anthracycline-naïve patients, ORR was $28.0 \%$ and mPFS 10.9 months (95\% Cl: 1.8-15.3). Imaging with ${ }^{64} \mathrm{Cu}$-labeled MM-302 visualized tumor-drug penetrance in tumors throughout the body, including the brain.

CONCLUSION: MM-302 monotherapy, in combination with trastuzumab, or trastuzumab plus cyclophosphamide, was well tolerated and showed promising efficacy. The selected phase $2 \mathrm{MM}-302$ dose was $30 \mathrm{mg} / \mathrm{m}^{2} \mathrm{plus} 6 \mathrm{mg} / \mathrm{kg}$ trastuzumab q3w.

British Journal of Cancer (2018) 119:1086-1093; https://doi.org/10.1038/s41416-018-0235-2

\section{BACKGROUND}

Human epidermal growth factor receptor 2 (HER2)-targeted anticancer therapies such as trastuzumab, pertuzumab, and adotrastuzumab emtansine (T-DM1) have transformed the treatment of HER2-positive advanced/metastatic breast cancer. ${ }^{1-3}$ Despite these improvements, the disease ultimately progresses, and new treatment strategies are required.

Anthracyclines, such as doxorubicin, are efficacious in the treatment of HER2-positive breast cancer. ${ }^{4-6}$ However, their use in early-stage trastuzumab-containing breast cancer regimens is limited by their adverse effects on the heart, ${ }^{2}$ and some studies show that a taxane-based regimen may have similar efficacy in the adjuvant and neoadjuvant settings. ${ }^{6,7}$ There is a strong need for efficacious regimens after taxane failure in the metastatic setting, and less toxic early-stage regimens. Compared with free doxorubicin, liposomal formulations of doxorubicin have less cardiac toxicity and fewer side effects. ${ }^{8-16}$ The introduction of targeted antibody conjugates has provided great promise to maximize cancer cell uptake while minimizing systemic toxicity. Targeted delivery of doxorubicin to HER2-overexpressing cells would allow use of one of the most effective chemotherapeutic agents while sparing the heart.

MM-302 is a HER2-targeted PEGylated antibody-liposomal doxorubicin conjugate that targets HER2overexpressing tumor cells, delivers doxorubicin to tumor cells, and limits exposure to healthy cells such as cardiomyocytes (Supplemental Fig. 1). ${ }^{17,18}$ In preclinical models of HER2-positive breast cancer, MM-302 demonstrated superior antitumor activity compared to both doxorubicin and PEGylated liposomal doxorubicin. ${ }^{18}$ MM302 and trastuzumab bind different epitopes of HER2, and the combination of MM-302 and trastuzumab demonstrated enhanced antitumor activity compared with either agent alone in HER2overexpressing

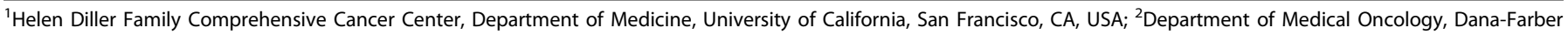

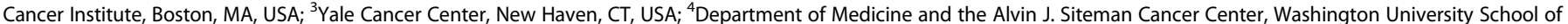

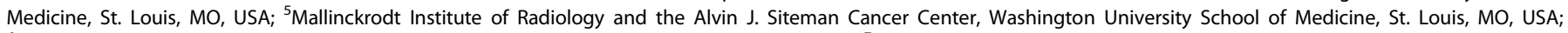

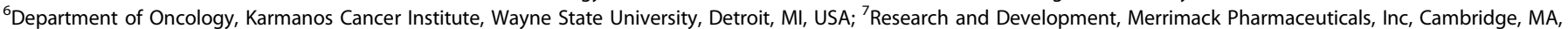
USA and ${ }^{8}$ Indiana University Melvin and Bren Simon Cancer Center, Indianapolis, IN, USA Correspondence: Pamela Munster (Pmunster@medicine.ucsf.edu) 
Table 1. Treatment administration and dose-limiting toxicity

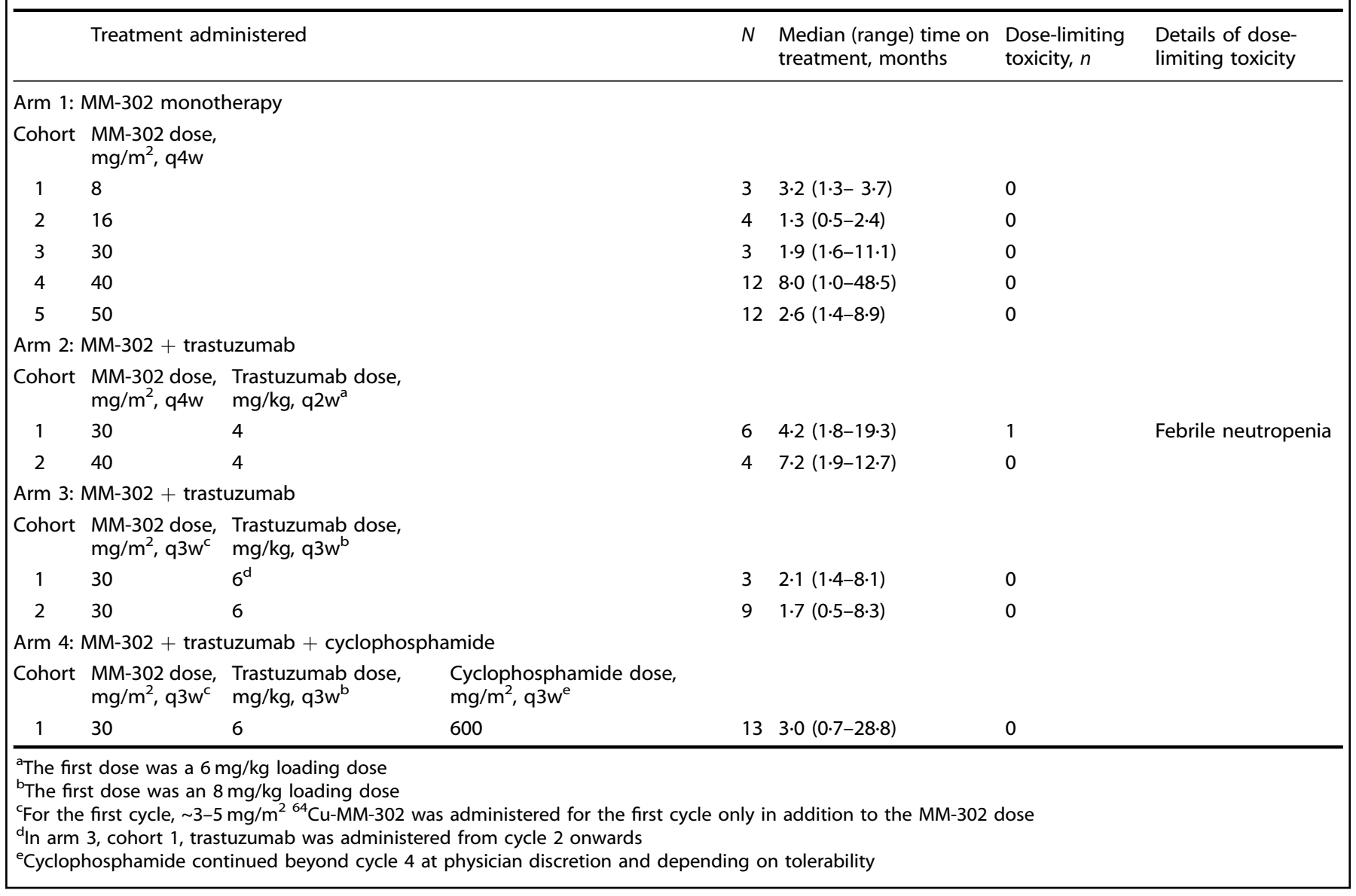

tumor xenograft models. ${ }^{12,19-21}$ Preclinical studies have also shown that cyclophosphamide pretreatment increased the delivery and antitumor activity of MM-302. ${ }^{22}$ These findings support clinical investigation of MM-302 in combination with trastuzumab and trastuzumab plus cyclophosphamide. Here we report the results from the first-in-human phase 1 study of MM302 in patients with advanced HER2-positive breast cancer.

\section{METHODS}

Study design

This phase 1, open-label, multicenter, dose-escalation study evaluated the safety, tolerability, and pharmacokinetics (PK) of MM-302 as a monotherapy (arm 1), in combination with trastuzumab (arm 2 and arm 3), or trastuzumab plus cyclophosphamide (arm 4) in patients with advanced HER2-positive breast cancer (ClinicalTrials.gov Identifier: NCT01304797).

The primary objectives were to assess safety and tolerability, and to determine the maximum tolerated dose (MTD) and phase 2 dose of MM-302 as a monotherapy, in combination with trastuzumab or trastuzumab plus cyclophosphamide. Secondary objectives were to describe dose-limiting toxicities (DLTs), objective response rate (ORR; confirmed complete response [CR] or partial response $[P R])$, clinical benefit rate $(C B R$; confirmed $C R$, PR or stable disease at 24 weeks), duration of response, progression-free survival (PFS), immunogenicity and PK of MM302 in patients enrolled in arm 1-4. Exploratory objectives investigated the correlation of clinical activity with levels of HER2 amplification or protein expression, and MM-302 biodistribution and tumor deposition.
The trial was conducted at five sites in the United States and was approved by the Institutional Review Board at each site. All patients provided written informed consent prior to study enrollment in accordance with the Declaration of Helsinki, the Code of Federal Regulations and local regulatory requirements.

Patients

Key inclusion criteria were: histologically or cytologically confirmed HER2-positive locally advanced/metastatic breast cancer; tumor amenable to biopsy; measurable disease per Response Evaluation Criteria in Solid Tumors (RECIST) version 1.1; age $\geq 18$ years; Eastern Cooperative Oncology Group (ECOG) performance status 0 or 1 ; left ventricular ejection fraction (LVEF) $\geq 50 \%$; adequate renal/hepatic function and bone marrow reserves. Patients with stable, treated central nervous system metastases were allowed.

Key exclusion criteria were: previous cumulative anthracycline total dose of $>300 \mathrm{mg} / \mathrm{m}^{2}$ doxorubicin equivalent; significant cardiovascular problems.

Treatment

MM-302 (Merrimack Pharmaceuticals, Cambridge, MA, USA) was administered by intravenous infusion over $60 \mathrm{~min}$ and the dose based on a doxorubicin hydrochloride equivalent basis. Patients were sequentially enrolled in one of four arms (Table 1). A standard ' $3+3$ ' dose escalation design was used starting with MM-302 monotherapy (arm 1; 8, 16, 30, 40, and $50 \mathrm{mg} / \mathrm{m}^{2}$ ) administered once every 4 weeks ( $q 4 \mathrm{w}$ ) with expansion cohorts at 40 and $50 \mathrm{mg} / \mathrm{m}^{2}$ (each containing 12 patients, respectively). Arm 2 was a combination dose escalation of intravenous MM-302 
(30 and $40 \mathrm{mg} / \mathrm{m}^{2} \mathrm{q} 4 \mathrm{w}$ ) plus intravenous trastuzumab $(4 \mathrm{mg} / \mathrm{kg}$ with $6 \mathrm{mg} / \mathrm{kg}$ loading) every 2 weeks (q2w). Arm 3 cohort 1 investigated MM-302 $\left(30 \mathrm{mg} / \mathrm{m}^{2}\right)$ on day 1 cycle 1 , initially in three patients; in subsequent cycles patients received trastuzumab $\left(6^{*}\right.$ or $8 \mathrm{mg} / \mathrm{kg}$ [*with $8 \mathrm{mg} / \mathrm{kg}$ loading] every 3 weeks [q3w]) on day 1 followed by MM-302 on day 6. After safety had been established in the first cohort, a larger expansion cohort (cohort 2) of nine patients investigated trastuzumab administered on cycle 1 day 1 , followed by MM-302 and ${ }^{64} \mathrm{Cu}-\mathrm{MM}-302$ on cycle 1 day 6 . During subsequent cycles, trastuzumab was administered $q 3 \mathrm{w}$ on day 1

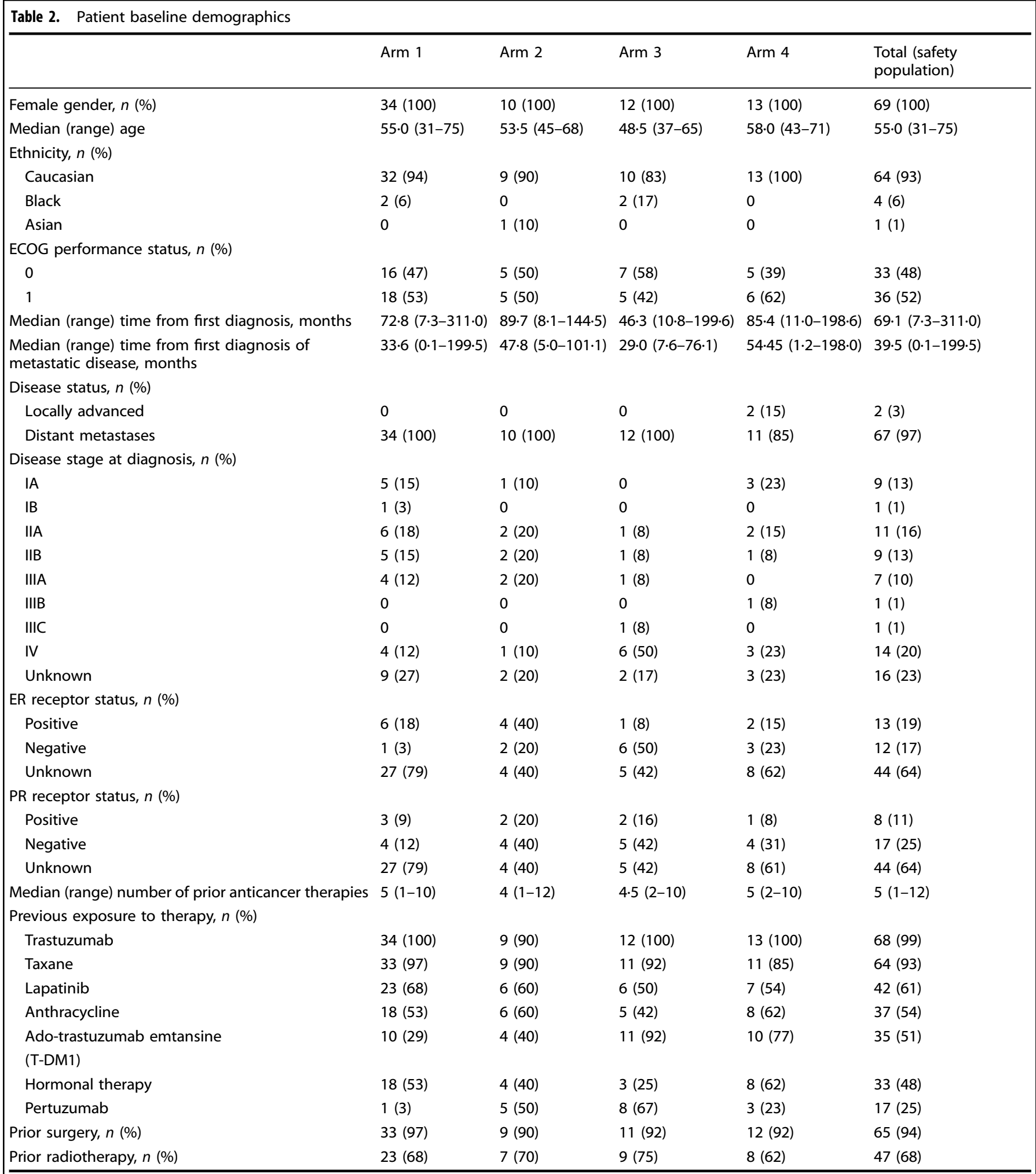


and a full therapeutic dose of non-radioactive MM-302 $\left(30 \mathrm{mg} / \mathrm{m}^{2}\right)$ was administered $\mathrm{q} 3 \mathrm{w}$ on day 6 . Similarly, arm 4 investigated intravenous cyclophosphamide $\left(450 \mathrm{mg} / \mathrm{m}^{2} \mathrm{q} 3 \mathrm{w}\right)$ and trastuzu$\mathrm{mab}(6 \mathrm{mg} / \mathrm{kg}$ after $8 \mathrm{mg} / \mathrm{kg}$ loading) on day 1 followed by MM$302\left(30 \mathrm{mg} / \mathrm{m}^{2} \mathrm{q} 3 \mathrm{w}\right)$ on day 6 in 13 patients. Cyclophosphamide administration in arm 4 continued beyond cycle 4 at physician discretion and depending on tolerability.

Following treatment with unlabeled MM-302, patients in arms 3 and 4 also received $337-432 \mathrm{MBq}{ }^{64} \mathrm{Cu}-\mathrm{MM}-302\left({ }^{64} \mathrm{Cu}-\mathrm{MM}-302\right)$, mass dosage $3-5 \mathrm{mg} / \mathrm{m}^{2}$, during cycle 1 , on the first day of $\mathrm{MM}$ 302 administration only, and then 2-3 positron emission tomography/computed tomography (PET/CT) scans were obtained immediately after administration and up to 2 days later to assess ${ }^{64} \mathrm{Cu}-\mathrm{MM}-302$ biodistribution and tumor deposition. ${ }^{23,24}$

Patients were treated until disease progression, unacceptable toxicity, or withdrawal of consent. A $25 \%$ dose reduction was permitted for any toxicity $\geq$ grade 3 that was considered related and/or significant by the investigator. Treatment could be paused for $\leq 56$ days to allow recovery from an adverse event $(A E)$; otherwise treatment was discontinued.

Patients experiencing an asymptomatic reduction in LVEF to $<50 \%$ or $>10 \%$ absolute drop from baseline could continue treatment at the discretion of the medical monitor, provided that cardiac function tests were performed prior to each MM-302 dose. Study treatment was discontinued permanently if LVEF did not improve or declined further.

From September 2012, all patients starting treatment received prophylactic premedication with $25-50 \mathrm{mg}$ diphenhydramine or equivalent (administered orally $\leq 30 \mathrm{~min}$ prior to $\mathrm{MM}-302$ dosing or intravenously immediately prior to MM-302 dosing) in cycle 1. Patients who tolerated the initial dose without an infusion reaction could discontinue premedication in subsequent cycles according to investigator judgment.

\section{Assessments}

DLTs were evaluated during cycle 1 of treatment and AEs were monitored throughout treatment and for 30 days after administration of the last study drug dose, according to the National Cancer Institute Common Terminology Criteria for Adverse Events ( $\mathrm{NCl} \mathrm{CTCAE}$ ) version 4.0.

Cardiac function was regularly monitored throughout the study. Electrocardiograms were obtained at screening, prior to each cycle, at study end, and at 30-day follow-up. Radionuclide ventriculography or echocardiography was obtained at screening, every 4 weeks for the first three cycles then every 8 weeks thereafter, at study end, and at 30-day follow-up.

Blood samples for PK analysis were collected immediately before and after MM-302 infusion, and at approximately 2, 4, 8, 24, 48 , and $72 \mathrm{~h}$ after the start of infusion, 8 days after treatment, at study termination, and at 30-day follow-up. Samples were analysed for total and encapsulated doxorubicin, as well as the anti-HER2 single chain variable fragment (scFv), F5. Analysis was performed comparing PK parameters to drug activity. Anti-drug antibody samples were collected before dosing and at the onset of any infusion reaction, at the resolution of the event, and at 28 days after the event.

ORR was assessed by RECIST version 1.1 at screening and every 8 weeks from first dose. CBR, median duration of response, and median PFS were calculated per dose cohort and for the overall population.

Tumor biopsy samples were collected $72 \mathrm{~h}$ after MM-302 dosing to measure HER2, microvessel density, and microscopic distribution of MM-302 within the tumor. A subgroup of patients received ${ }^{64} \mathrm{Cu}-\mathrm{MM}-302$ to map biodistribution and tumor deposition, under a companion imaging protocol described elsewhere. ${ }^{23,24}$

Statistical analysis

Patients who received at least a partial infusion of MM-302 during this period were included in the safety and efficacy population. Efficacy was also reported separately for patients who received $\geq 30 \mathrm{mg} / \mathrm{m}^{2} \mathrm{MM}-302$. Safety, efficacy, and PK parameters were summarized using descriptive statistics for continuous variables and frequency distributions for categorical variables.

Table 3. Most common treatment-emergent adverse events all grade occurring in $>20 \%$ of patients in the overall population and grade $3 / 4$ adverse events occurring in more than two patients in any treatment arm (safety population)

\begin{tabular}{|c|c|c|c|c|c|c|c|c|c|c|}
\hline Fatigue & $21(62)$ & 0 & $4(40)$ & 0 & $6(50)$ & $1(11)$ & $3(23)$ & $1(8)$ & $34(49)$ & $2(3)$ \\
\hline Nausea & $19(56)$ & 0 & $2(20)$ & 0 & $7(58)$ & 0 & $6(46)$ & 0 & $34(49)$ & 0 \\
\hline Decreased appetite & $13(38)$ & 0 & $2(20)$ & 0 & $3(25)$ & 0 & $2(15)$ & 0 & $20(29)$ & 0 \\
\hline Vomiting & $9(27)$ & 0 & $1(10)$ & 0 & $4(33)$ & 0 & $5(39)$ & 0 & $19(28)$ & 0 \\
\hline Constipation & $7(21)$ & 0 & $2(20)$ & 0 & $3(25)$ & 0 & $4(31)$ & 0 & $16(23)$ & 0 \\
\hline Stomatitis & $8(24)$ & 0 & $4(40)$ & 0 & $2(17)$ & 0 & $2(15)$ & 0 & $16(23)$ & 0 \\
\hline Dyspnea & $5(15)$ & $2(6)$ & $2(20)$ & 0 & $5(42)$ & 0 & $4(31)$ & 0 & $16(23)$ & 0 \\
\hline Neutropenia & $9(27)$ & $6(18)$ & 0 & 0 & $3(25)$ & 0 & $3(23)$ & $1(8)$ & $15(22)$ & $7(10$ \\
\hline Rash & $9(27)$ & 0 & $1(10)$ & 0 & $1(8)$ & 0 & $3(23)$ & 0 & $14(20)$ & 0 \\
\hline
\end{tabular}




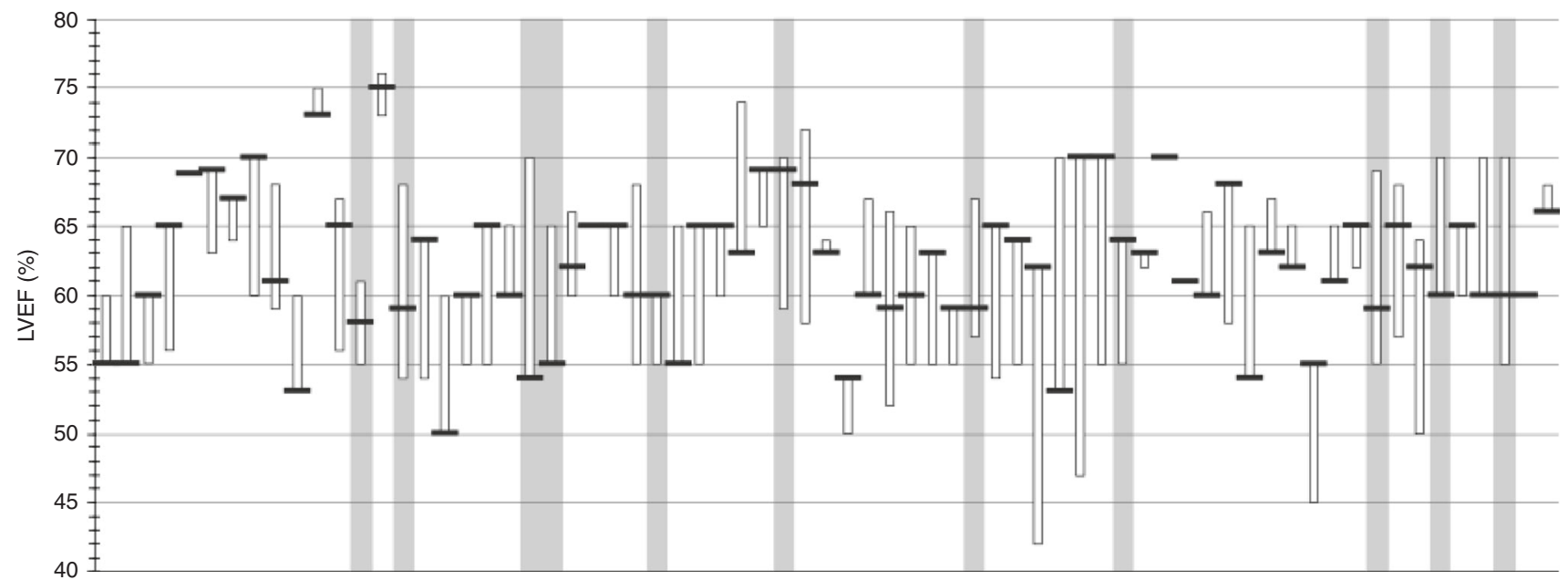

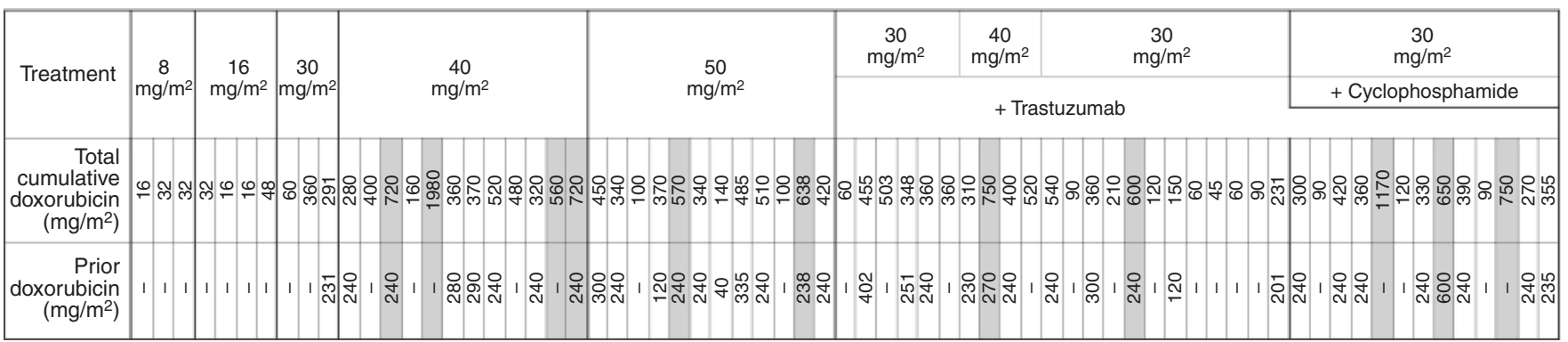

Fig. 1 Cardiac safety. Left ventricular ejection fraction (LVEF) is shown by patient (black horizontal line denotes baseline value, box denotes range over treatment period). Treatment received, including dose of $\mathrm{MM}$-302, is shown below graph for each patient as well as cumulative doxorubicin exposure. Grey highlighting shows the 11 patients with cumulative anthracycline exposure $>550 \mathrm{mg} / \mathrm{m}^{2}$

\section{RESULTS}

Seventy-three patients were enrolled between 25 July 2011 and 24 June 2014, 69 of whom received study treatment in four arms with MM-302 either alone or in combination with trastuzumab and cyclophosphamide (Table 1 and Supplemental Fig. 2). Patient demographics and characteristics are described in Table 2. At data cutoff (18 August 2016), study treatment was ongoing for one patient receiving MM-302 monotherapy and one patient receiving MM-302 plus trastuzumab and cyclophosphamide (Supplemental Fig. 2).

Patients remained on study treatment for a median of 3.0 months (range: $0.5-48.5$ ). Of note, as of data cutoff, one patient had received $>49$ cycles of $40 \mathrm{mg} / \mathrm{m}^{2} \mathrm{MM}-302 \mathrm{q} 4 \mathrm{w}$ for a cumulative dose of $1960 \mathrm{mg} / \mathrm{m}^{2} \mathrm{MM}-302$ and was continuing to receive study treatment.

The most common AEs of any grade were fatigue and nausea (MM-302 monotherapy plus trastuzumab), or nausea and vomiting (MM-302 plus trastuzumab and cyclophosphamide) (Table 3). Specific AEs for doxorubicin, e.g., cardiac toxicity, were followed as AEs of special interest (Supplemental Table 1). Grade 3 or 4 treatment-emergent AEs occurred in 24/69 patients (Table 3); neutropenia was the most common in $6 / 34$ (18\%) patients receiving monotherapy and $1 / 13(8 \%)$ patients receiving $\mathrm{MM}$ 302 plus trastuzumab. Median time to absolute neutrophil count nadir was 23 days. The highest incidence of grade 3/4 AEs was seen with $50 \mathrm{mg} / \mathrm{m}^{2} \mathrm{MM}-302$ monotherapy ( $\left.n=5 / 12 ; 42 \%\right)$. As this occurred beyond cycle 1 , neutropenia did not meet the criteria of DLT. Neutropenia was primarily observed in patients receiving higher dose intensities (>10 $\mathrm{mg} / \mathrm{m}^{2} /$ week). In addition, grade 3/4 neutropenia was observed only in patients previously treated with an anthracycline (Supplemental Figure 4).

Seventeen patients had $\geq 1$ dose interruption, and of these, 15 patients had $\geq 1$ dose interruption due to infusion reaction. Eight patients discontinued treatment due to an $\operatorname{AE}(n=1$ for each of convulsion; aphasia/face edema; peripheral sensory neuropathy; cardiac failure [see below]; neutropenia; hypoxia; thrombocytopenia; back pain/dyspnea/infusion-related reaction). Three patients experienced $\geq 1$ treatment-related serious AE (anemia/neutropenia/thrombocytopenia; palmar-plantar erythrodysesthesia syndrome; febrile neutropenia). Only one DLT (febrile neutropenia) was observed in a patient treated with $30 \mathrm{mg} / \mathrm{m}^{2} \mathrm{MM}-302 \mathrm{q} 4 \mathrm{w}$ plus $4 \mathrm{mg} / \mathrm{kg}$ trastuzumab q2w (Table 1). There were no treatment-related deaths. The MTD was not defined in this trial; however, post-cycle 1 neutropenia prompted the recommendation of a phase 2 dose of $30 \mathrm{mg} / \mathrm{m}^{2} \mathrm{q} 3 \mathrm{w}$ or $40 \mathrm{mg} / \mathrm{m}^{2} \mathrm{q} 4 \mathrm{w}$.

There were no reported cardiac AEs $\geq$ grade 2 with MM-302 monotherapy, and cardiac AEs were infrequent in the MM-302 combination arms with no serious AEs. Six patients on the combination arms had a protocol-defined asymptomatic decline in LVEF defined as (1) a post-baseline measurement $<50 \%$, or 2) a post-baseline measurement that was a reduction of $>10$ absolute percentage points from baseline (Fig. 1): three patients had LVEF $<50 \%$; four patients had reversible declines; one patient had a decline to $47 \%$ noted at off-study assessment; one patient (receiving $30 \mathrm{mg} / \mathrm{m}^{2} \mathrm{MM}-302$ and $6 \mathrm{mg} / \mathrm{kg}$ trastuzumab q3w) was observed twice to have a range of $45-50 \%$ LVEF (unchanged off-study). This patient discontinued treatment because of LVEF decline. Eleven patients had lifetime cumulative anthracycline exposure $>550 \mathrm{mg} / \mathrm{m}^{2}$ with no protocol-defined LVEF changes (Fig. 1).

Total doxorubicin, encapsulated doxorubicin, and anti-HER scFv F5 plasma concentrations were measured (Supplemental Fig. 3) and pharmacokinetic parameters summarized in Supplemental Table 2. The terminal half-lives at the first cycle are estimated using non-compartmental analysis to be $47 \cdot 3,44 \cdot 8$, and $63.3 \mathrm{~h}$ for total doxorubicin, encapsulated doxorubicin, and F5, and these values increased by $\sim 15 \%$ in subsequent cycles. Measurements of total and encapsulated doxorubicin indicated that the two 

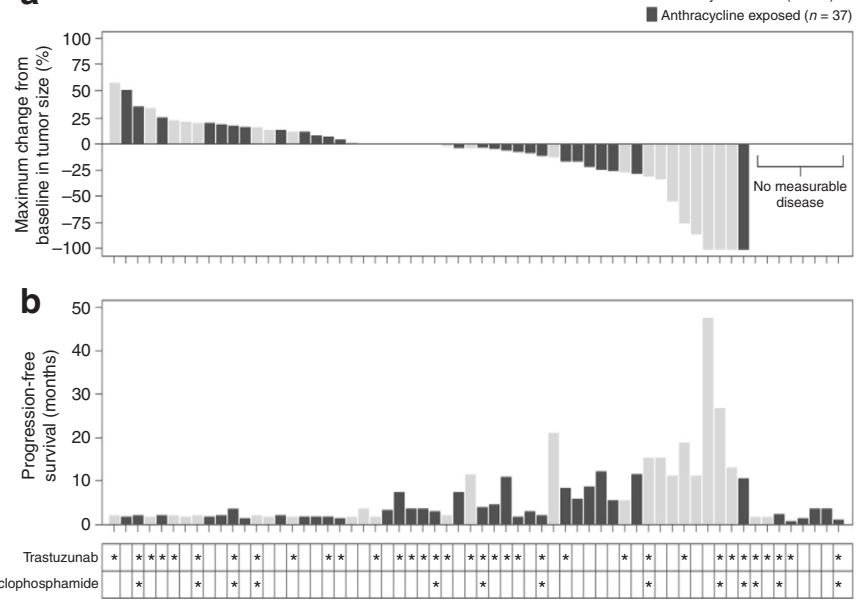

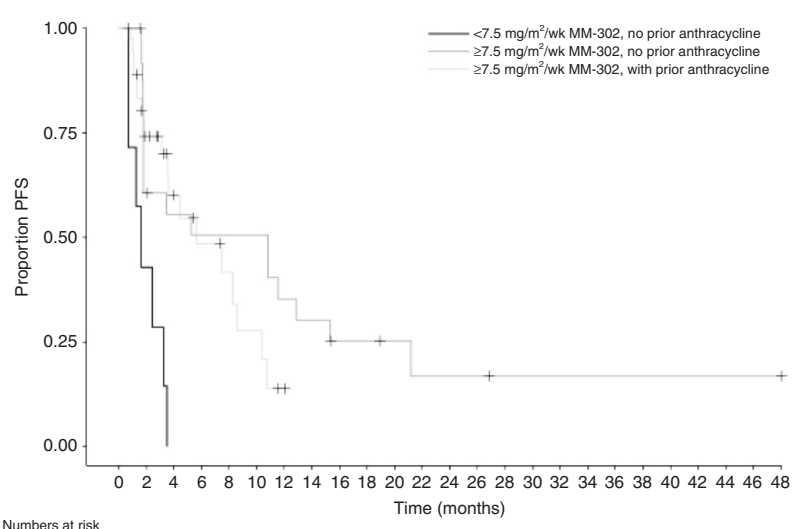

Nrimbers at risk $\begin{array}{llllllllllllllllllllllllllllll}7 & 3 & 0 & 0 & 0 & 0 & 0 & 0 & 0 & 0 & 0 & 0 & 0 & 0 & 0 & 0 & 0 & 0 & 0 & 0 & 0 & 0 & 0 & 0 & 0\end{array}$

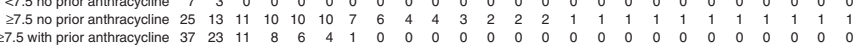

Fig. 2 a Maximum change from baseline in tumor size (sum of target lesions) and progression-free survival (PFS). b Maximum change from baseline in tumor size (sum of target lesions) and PFS in patients treated with MM-302 $\geq 30 \mathrm{mg} / \mathrm{m}^{2}$ (safety population). c Kaplan-Meier graph of PFS in subgroups split by average MM-302 dose intensity and prior anthracycline status $\left(<7.5 \mathrm{mg} / \mathrm{m}^{2} / \mathrm{week}, \geq 7.5 \mathrm{mg} / \mathrm{m}^{2} / \mathrm{week}\right.$ with and without prior anthracycline)

a

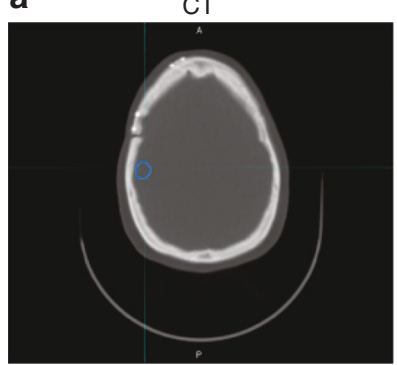

C

C PET

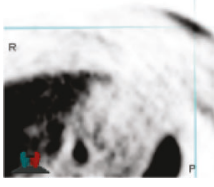

e

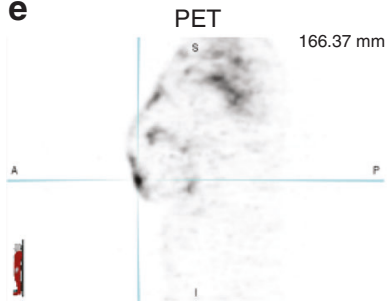

b

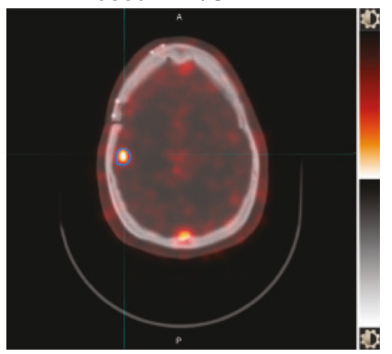

d

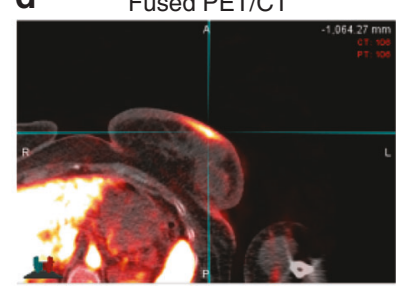

f

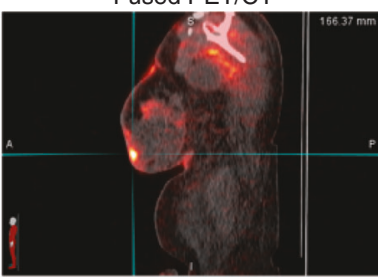

g

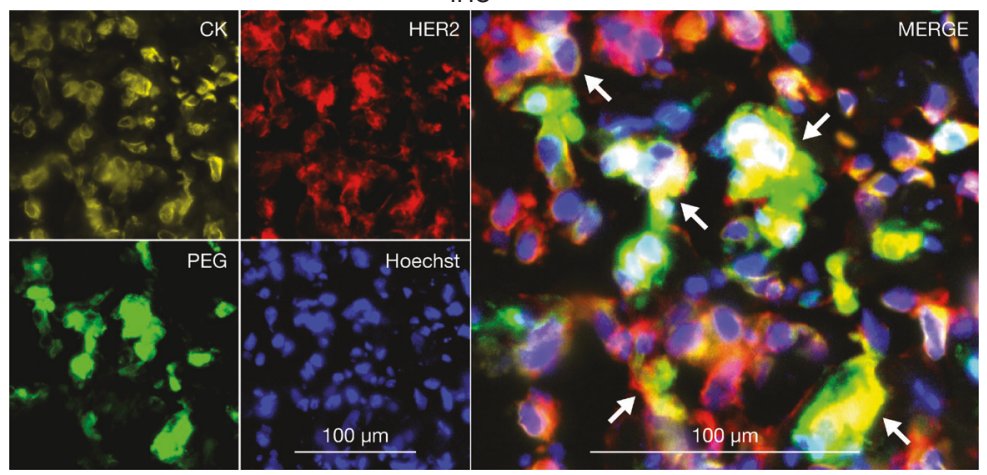

Fig. 3 a, b Are representative axial CT and PET images in two patients with HER2-positive metastatic breast cancer at 24-h post-administration of ${ }^{64} \mathrm{Cu}-\mathrm{MM}-302$, respectively, of the head. Brain lesion (as confirmed by MRI at screening) is indicated by the blue contour and is shown to have significant uptake of ${ }^{64} \mathrm{Cu}-\mathrm{MM}-302$ as seen in (b). c-f Show ${ }^{64} \mathrm{Cu}-\mathrm{MM}-302$ deposition in a skin lesion in another patient. c-f Are representative axial and sagittal views, respectively, of the skin lesion (as indicated by the cyan cross hair marker). g Immunofluorescence images (20x) of a day 3 liver biopsy of a study patient (IHC 3+ and FISH positive on FFPE) treated with $30 \mathrm{mg} / \mathrm{m}^{2} \mathrm{MM}-302 \mathrm{q} 4 \mathrm{w}$. The biopsy was stained for cytokeratin (CK, yellow) to identify tumor cells, HER2 (red), PEG (green) to identify MM-302, and nuclei with Hoechst (blue) (left four panels). Merged images (right panel) show clusters of HER2-positive tumor cells with internalized liposomes (arrows). CT computed tomography, HER2 human epidermal growth factor receptor 2, MRI magnetic resonance imaging, PET positron emission tomography, FFPE formalin-fixed paraffin-embedded, FISH fluorescence in situ hybridization, HER2 human epidermal growth factor receptor 2, IHC immunohistochemistry, PEG polyethylene glycol, q4w every 4 weeks 
analytes are highly correlated; $98 \%$ doxorubicin remained encapsulated in samples collected up to 1 week. Area under the curve (AUC) and maximum concentration $\left(C_{\max }\right)$ of total and encapsulated doxorubicin and F5 increased proportionally with dose.

In four patients, positively confirmed anti-drug antibodies (ADAs) non-specific to MM-302 were detected at baseline, prior to any MM-302 treatment, and were also detected post-baseline. In two other patients, ADAs were detected post-baseline at cycle 2 and 6 and titer levels remained $\leq 40$ relative luminescence units (RLU); both had negative ADAs at study end.

The patient population in this trial was heavily pretreated, with a median of five prior therapies and $99 \%$ of patients had received prior trastuzumab. Overall, ORR was $12 \%(n=8 / 69 ; 95 \% \mathrm{Cl}: 5 \cdot 1$, $21.6)$ and CBR was $28 \%(n=19 / 69 ; 95 \% \mathrm{Cl}: 17 \cdot 5,39 \cdot 6)$. No responses were observed in patients receiving $<30 \mathrm{mg} / \mathrm{m}^{2}$ of MM302. Median PFS was 4.4 months (95\% Cl: $3 \cdot 1,8 \cdot 6)$.

In patients receiving $\geq 30 \mathrm{mg} / \mathrm{m}^{2}$ MM-302 ( $n=62: 27$ monotherapy; 22 plus trastuzumab; 13 plus trastuzumab/cyclophosphamide), ORR was $13 \%$ ( $n=8 / 62$; two CR and six PR) (Fig. 2a), CBR was $30.6 \%(n=19 / 62)$ and median PFS was 7.4 months $(95 \%$ $\mathrm{Cl}$ : 3.5-10.9) (Fig. 2b). Among patients receiving $\geq 30 \mathrm{mg} / \mathrm{m}^{2} \mathrm{MM}-$ 302 , in the anthracycline-exposed group $(n=37)$, ORR was $3 \%(n$ $=1 / 37)$, CBR was $24 \%(n=9 / 37)$ and median PFS 5.6 months $(95 \%$ $\mathrm{Cl}: 3 \cdot 5-8 \cdot 6)$. In the anthracycline-naïve subgroup $(n=25)$, ORR was $28 \%(n=7 / 25)$, CBR was $40 \%(n=10 / 25)$ and median PFS was 10.9 months (95\% Cl: 1.8-15.3) (Fig. 2a, b). A relationship was observed between clinical efficacy and dose, suggesting that a greater reduction in tumor burden was observed at doses $\geq 30$ $\mathrm{mg} / \mathrm{m}^{2}$ (Fig. $2 \mathrm{c}$ and Supplementary Figure 5).

PET/CT following administration of ${ }^{64} \mathrm{Cu}-\mathrm{MM}-302$ revealed diverse locations of tumor MM-302 uptake in different patients, including skin, breast, liver, and brain (Fig. 3a-f), and with different lesion intensities both intra- and interpatient. ${ }^{23}$ High levels of MM-302 in the blood and liver at the time points used for imaging were consistent with the anticipated biodistribution for a PEGylated liposomal agent. Furthermore, HER2-mediated delivery of MM-302 to HER2-overexpressing tumor cells was confirmed in multiple evaluable biopsies post-MM-302 treatment by co-localization of antiPEG, anti-HER2, and anti-cytokeratin antibodies (Fig. 3g).

\section{DISCUSSION}

In this phase 1, open-label, dose-escalation study, MM-302 was well tolerated and active in patients with HER2-positive advanced/ metastatic breast cancer. The study population was heavily pretreated with a median of five prior regimens for metastatic disease. The main study objective was to determine the safety of MM-302 as a monotherapy and in combination with trastuzumab and cyclophosphamide. Consistent with the nature of a phase 1 dose escalation study, conclusions on efficacy are confounded by the heterogeneous doses and schedules of MM-302 used and the multitude of monotherapy and combination therapy cohorts. Nonetheless, there is notable lasting activity without cumulative toxicity.

MM-302 was designed to target delivery of doxorubicin to tumors via anti-HER2-directed antibodies. Owing to the low level of HER2-expression on cardiomyocytes, preclinical studies with MM-302 show no uptake into human stem cell-derived cardiomyocytes and no effect on cell viability. ${ }^{18}$ However, to safeguard against cardiac toxicity, effects on the heart were monitored very closely. No cardiac AEs were observed in patients treated with MM-302 monotherapy. With MM-302 in combination with trastuzumab, with or without cyclophosphamide, cardiac AEs were infrequent with no serious AEs.

MM-302 activity correlated with AUC and, in general, higher activity was observed at doses $\geq 10 \mathrm{mg} / \mathrm{m}^{2} /$ week $\left(30 \mathrm{mg} / \mathrm{m}^{2} \mathrm{q} 3 \mathrm{w}\right.$ and $40 \mathrm{mg} / \mathrm{m}^{2} \mathrm{q} 4 \mathrm{w}$ ) (Fig. 3 and Supplemental Figure 5). Although
MTD was not reached at $50 \mathrm{mg} / \mathrm{m}^{2} \mathrm{q} 4 \mathrm{w}\left(12.5 \mathrm{mg} / \mathrm{m}^{2} /\right.$ week), dose intensity of $10 \mathrm{mg} / \mathrm{m}^{2} /$ week was also active and had fewer cumulative incidences of neutropenia. Based on phase 1 results, the recommended phase 2 dose was $30 \mathrm{mg} / \mathrm{m}^{2}$ q3w MM-302 (10 $\mathrm{mg} / \mathrm{m}^{2} /$ week) in combination with trastuzumab $6 \mathrm{mg} / \mathrm{kg} \mathrm{q} 3 \mathrm{w}$ (with $8 \mathrm{mg} / \mathrm{kg}$ loading).

The observed activity of MM-302 is promising. Median PFS of 7.4 months with $\geq 30 \mathrm{mg} / \mathrm{m}^{2} \mathrm{MM}-302$ monotherapy, in combination with trastuzumab or with trastuzumab and cyclophosphamide, compares favourably with recent late-stage HER2-positive clinical trials that demonstrated median PFS of 3.3 months in patients treated with the physician's choice. ${ }^{25}$ Furthermore, in the present study, the observed PFS and ORR in patients not previously exposed to an anthracycline were 10.9 months and $28 \%$, respectively. Multiple studies of liposomal doxorubicin have observed similar trends, with improved activity in patients not previously exposed to an anthracycline compared to those previously exposed. ${ }^{12,20,21}$ Additionally, grade 3 palmar-plantar erythrodysesthesia with MM-302 $(n=1 / 69 ; 1 \%)$ was less frequent than that reported for the combination of liposomal doxorubicin plus trastuzumab ( $n=9 / 30 ; 30 \%$; respectively). ${ }^{9}$

Exploratory analyses of tumor biopsies support a HER2targeting mechanism for MM-302 consistent with preclinical data. Tumor deposition data using ${ }^{64} \mathrm{Cu}-\mathrm{MM}-302$ PET/CT revealed the potential of MM-302 to be delivered to difficult metastatic locations such as brain metastases. ${ }^{23}$

In summary, these data suggest that MM302 monotherapy, in combination with trastuzumab or trastuzumab and cyclophosphamide has a manageable safety profile and promising clinical activity in patients with advanced HER2-positive breast cancer. MM-302 $30 \mathrm{mg} / \mathrm{m}^{2}$ in combination with $6 \mathrm{mg} / \mathrm{kg}$ trastuzumab every 3 weeks was selected as the recommended phase 2 study dose. This study is now completed, and further clinical studies are under discussion.

\section{ACKNOWLEDGEMENTS}

We thank all the patients, caregivers, and investigators who participated in this study. We also thank Dr. Ty McClure, who provided statistical analysis of the study, and Dr. Helen Lee, who was instrumental in carrying out the ${ }^{64} \mathrm{Cu}-\mathrm{MM}-302$ imaging analysis. We thank Translational Drug Development (TD2), who provided contract research organization support for this study funded by Merrimack Pharmaceuticals. This study used the Siteman Cancer Center Imaging and Response Assessment Core supported in part by an GR NCI Cancer Center Support Grant (\#P30 CA91842).

\section{AUTHOR CONTRIBUTIONS}

P.M. contributed to study design, recruited patients, analyzed and interpreted data, and contributed to writing and approval of the manuscript. C.M. contributed to data collection and contributed to writing and approval of the manuscript. P.L. contributed to study design, recruited patients, collected data, analyzed and interpreted data, and contributed to writing and approval of the manuscript. I.E.K. contributed to study design, recruited patients, and contributed to writing and approval of the manuscript. B.A.S. contributed to study design, collected data, recruited patients, analyzed and interpreted data, and contributed to writing and approval of the manuscript. A.F.S. contributed to study design, collected data, recruited patients, analyzed and interpreted data, and contributed to writing and approval of the manuscript. I.M. contributed to analysis, interpretation of data, and contributed to writing and approval of the manuscript. J.R. contributed to study design, analysis, interpretation of data, and contributed to writing and approval of the manuscript. K.C. contributed to study design and contributed to writing and approval of the manuscript. B.S.H. contributed to study design, analysis, interpretation of data, and contributed to writing and approval of the manuscript. B.A. contributed to analysis, interpretation of data, and contributed to writing and approval of the manuscript. E.G. contributed to collection, analysis and interpretation of data, and contributed to writing and approval of the manuscript. V.M. contributed to study design and contributed to writing and approval of the manuscript. K.D.M. contributed to study design, recruited patients, and contributed to writing and approval of the manuscript. T.J.W. contributed to study design, analysis, interpretation of data, and approval of the manuscript. All authors participated in the review and writing of the manuscript and gave final approval to submit for publication. 


\section{ADDITIONAL INFORMATION}

Supplementary information is available for this paper at https://doi.org/10.1038/ s41416-018-0235-2.

Ethics approval and consent to participate: The study protocol and its amendments were approved by the Independent Ethics Committees and/or Institutional Review Boards (Dana Farber Cancer Institute, Indiana University Health Melvin and Bren Simon Cancer Center, Wayne State University Department of Oncology, Karmanos Cancer Institute, University of California, San Francisco, Washington University School of Medicine). The study complied with recommendations of the 18th World Health Congress (Helsinki, 1964) and all applicable amendments. The study also complied with the laws and regulations, as well as any applicable guidelines, in the United States (the only country in which the study was conducted).

Funding: This trial was funded by Merrimack Pharmaceuticals, Inc. Merrimack Pharmaceuticals had roles in the study design; collection, analysis, and interpretation of data; writing of the report and decision to submit paper for publication. The corresponding author had full access to all the data in the study and had final responsibility for the decision to submit for publication.

Consent for publication: Not applicable. There is nothing to consent.

Competing interests: P.M. has nothing to declare. C.M.'s institution received financial support from Merrimack Pharmaceuticals to conduct this trial. P.L.'s former institution received financial support from Merrimack Pharmaceuticals to conduct this trial. Dr LoRusso has provided consultancy for: Agios, Alexion, Ariad, GenMab, Glenmark, Halozyme, Menarini, Novartis, Roche-Genentech, Genentech, CytomX, Omniox, and Ignyta. B.A.S. has received consulting fees from Merrimack Pharmaceuticals and his institution received financial support from Merrimack Pharmaceuticals to conduct this trial. Dr Siegel also received grants from the National Cancer Institute, and personal fees from Beacon Biosciences during the conduct of the study. AFS received consulting fees from Merrimack Pharmaceuticals and his institution received financial support from Merrimack Pharmaceuticals to conduct this trial. IM was an employee of Merrimack at the time of the study, and is a consultant for Ipsen Biosciences outside of the submitted work. J.R. was an employee of Merrimack at the time of the study. K. C. was an employee of Merrimack at the time of the study. B.S.H. was an employee of Merrimack at the time of the study. B.A. is an employee of Merrimack. E.G. was an employee of Merrimack at the time of the study. V.M. was an employee of Merrimack at the time of the study, is an equity owner in L.E.A.F. Pharmaceuticals LLC outside of the submitted work. In addition, Dr Moyo has a patent null pending. K.D.M. received financial support to her institution from Merrimack Pharmaceuticals to conduct this trial. T.J.W. was an employee of Merrimack at the time of the study.

Note: This work is published under the standard license to publish agreement. After 12 months the work will become freely available and the license terms will switch to a Creative Commons Attribution 4.0 International (CC BY 4.0).

\section{REFERENCES}

1. Baselga, J. et al. Pertuzumab plus trastuzumab plus docetaxel for metastatic breast cancer. N. Engl. J. Med. 366, 109-119 (2012).

2. Slamon, D. J. et al. Use of chemotherapy plus a monoclonal antibody against HER2 for metastatic breast cancer that overexpresses HER2. N. Engl. J. Med. 344, 783-792 (2001)

3. Verma, S. et al. Trastuzumab emtansine for HER2-positive advanced breast cancer. N. Engl. J. Med. 367, 1783-1791 (2012).

4. Giordano, S. H., Lin, Y. L., Kuo, Y. F., Hortobagyi, G. N. \& Goodwin, J. S. Decline in the use of anthracyclines for breast cancer. J. Clin. Oncol. 30, 2232-2239 (2012).

5. Montemurro, F. et al. Underuse of anthracyclines in women with HER-2+ advanced breast cancer. Oncologist 15, 665-672 (2010).

6. Slamon, D. et al. Adjuvant trastuzumab in HER2-positive breast cancer. N. Engl. J. Med. 365, 1273-1283 (2011).
7. Schneeweiss, A. et al. Pertuzumab plus trastuzumab in combination with standard neoadjuvant anthracycline-containing and anthracycline-free chemotherapy regimens in patients with HER2-positive early breast cancer: a randomized phase II cardiac safety study (TRYPHAENA). Ann. Oncol. 24, 2278-2284 (2013).

8. Baselga, J. et al. Phase III trial of nonpegylated liposomal doxorubicin in combination with trastuzumab and paclitaxel in HER2-positive metastatic breast cancer. Ann. Oncol. 25, 592-598 (2014).

9. Chia, S. et al. Pegylated liposomal doxorubicin and trastuzumab in HER-2 overexpressing metastatic breast cancer: a multicenter phase II trial. J. Clin. Oncol. 24, 2773-2778 (2006).

10. Christodoulou, C. et al. Trastuzumab combined with pegylated liposomal doxorubicin in patients with metastatic breast cancer. phase II Study of the Hellenic Cooperative Oncology Group (HeCOG) with biomarker evaluation. Oncology 76 275-285 (2009).

11. Gill, S. E. et al. Continuing routine cardiac surveillance in long-term use of pegylated liposomal doxorubicin: is it necessary? Gynecol. Oncol. 129, 544-547 (2013).

12. Keller, A. M. et al. Randomized phase III trial of pegylated liposomal doxorubicin versus vinorelbine or mitomycin $C$ plus vinblastine in women with taxanerefractory advanced breast cancer. J. Clin. Oncol. 22, 3893-3901 (2004).

13. O'Brien, M. E. et al. Reduced cardiotoxicity and comparable efficacy in a phase III trial of pegylated liposomal doxorubicin $\mathrm{HCl}$ (CAELYX/Doxil) versus conventional doxorubicin for first-line treatment of metastatic breast cancer. Ann. Oncol. 15 440-449 (2004).

14. Safra, T. et al. Pegylated liposomal doxorubicin (doxil): reduced clinical cardiotoxicity in patients reaching or exceeding cumulative doses of $500 \mathrm{mg} / \mathrm{m} 2$. Ann. Oncol. 11, 1029-1033 (2000).

15. Sparano, J. A. et al. Pegylated liposomal doxorubicin plus docetaxel significantly improves time to progression without additive cardiotoxicity compared with docetaxel monotherapy in patients with advanced breast cancer previously treated with neoadjuvant-adjuvant anthracycline therapy: results from a randomized phase III study. J. Clin. Oncol. 27, 4522-4529 (2009).

16. Wolff, A. C. et al. Phase II trial of pegylated liposomal doxorubicin plus docetaxel with and without trastuzumab in metastatic breast cancer: Eastern Cooperative Oncology Group trial E3198. Breast Cancer Res Treat. 121, 111-120 (2010).

17. Hendriks, B. S. et al. Impact of tumor HER2/ERBB2 expression level on HER2targeted liposomal doxorubicin-mediated drug delivery: multiple low-affinity interactions lead to a threshold effect. Mol. Cancer Ther. 12, 1816-1828 (2013).

18. Reynolds, J. G. et al. HER2-targeted liposomal doxorubicin displays enhanced anti-tumorigenic effects without associated cardiotoxicity. Toxicol. Appl. Pharmacol. 262, 1-10 (2012).

19. Espelin, C. W., Leonard, S. C., Geretti, E., Wickham, T. J. \& Hendriks, B. S. Dual HER2 targeting with trastuzumab and liposomal-encapsulated doxorubicin (MM-302) demonstrates synergistic antitumor activity in breast and gastric cancer. Cancer Res. 76, 1517-1527 (2016).

20. Fiegl, M. et al. Single-agent pegylated liposomal doxorubicin (PLD) in the treatment of metastatic breast cancer: results of an Austrian observational trial. BMC Cancer 11, 373 (2011).

21. Huober, J. et al. A multicentric observational trial of pegylated liposomal doxorubicin for metastatic breast cancer. BMC Cancer 10, 2 (2010).

22. Geretti, E. et al. Cyclophosphamide-mediated tumor priming for enhanced delivery and antitumor activity of HER2-targeted liposomal doxorubicin (MM302). Mol. Cancer Ther. 14, 2060-2071 (2015).

23. Lee, H. et al. 64Cu-MM-302 Positron emission tomography quantifies variability of enhanced permeability and retention of nanoparticles in relation to treatment response in patients with metastatic breast cancer. Clin. Cancer Res. 23, 4190-4202 (2017).

24. Lee, H. et al. A gradient-loadable (64)Cu-chelator for quantifying tumor deposition kinetics of nanoliposomal therapeutics by positron emission tomography. Nanomedicine 11, 155-165 (2015).

25. Krop, l. E. et al. Trastuzumab emtansine versus treatment of physician's choice for pretreated HER2-positive advanced breast cancer (TH3RESA): a randomised, open-label, phase 3 trial. Lancet Oncol. 15, 689-699 (2014). 